Proceedings

\title{
Incompatibility in Multi-Parameter Quantum Metrology with Fermionic Gaussian States ${ }^{\dagger}$
}

\author{
Angelo Carollo ${ }^{1,2, *(\mathbb{D})}$, Bernardo Spagnolo ${ }^{1,2,3}$ (1) and Davide Valenti ${ }^{1,4}$ (i) \\ 1 Department of Physics and Chemistry "Emilio Segré", Group of Interdisciplinary Theoretical Physics, \\ Palermo University and CNISM, Viale delle Scienze, Ed. 18, I-90128 Palermo, Italy \\ 2 Radiophysics Department, Lobachevsky State University of Nizhni Novgorod, 23 Gagarin Avenue, \\ Nizhni Novgorod 603950, Russia \\ 3 Istituto Nazionale di Fisica Nucleare, Sezione di Catania, Via S. Sofia 64, I-95123 Catania, Italy \\ 4 Istituto di Biomedicina ed Immunologia Molecolare (IBIM) “Alberto Monroy”, CNR, Via Ugo La Malfa 153, \\ I-90146 Palermo, Italy \\ * Correspondence: angelo.carollo@unipa.it \\ + Presented at the 11th Italian Quantum Information Science conference (IQIS2018), Catania, Italy, \\ 17-20 September 2018.
}

Published: 31 July 2019

check for updates

\begin{abstract}
In this article we derive a closed form expression for the incompatibility condition in multi-parameter quantum metrology when the reference states are Fermionic Gaussian states. Together with the quantum Fisher information, the knowledge of the compatibility condition provides a way of designing optimal measurement strategies for multi-parameter quantum estimation. Applications range from quantum metrology with thermal states to non-equilibrium steady states with Fermionic and spin systems.
\end{abstract}

Keywords: quantum metrology; Fermionic Gaussian state; quantum geometric information

\section{Introduction}

Estimation theory is the discipline that studies the accuracy by which a given set of physical parameters can be evaluated. When the parameters to be estimated belongs to an underlying quantum physical system one falls in the realm of quantum estimation theory, or quantum metrology [1]. Quantum parameter estimation finds applications in a wide variety of fields, from fundamental physics [2-6], to gravitational wave interferometry $[7,8]$, thermometry $[9,10]$, spectroscopy $[11,12]$, imaging [13-15], to name a few. Exploiting remarkable features of quantum systems as probes may give an edge over the accuracy of classical parameter estimation. Exploring this possibility plays a pivotal role in the current development of quantum technology [16-25]. In multi-parameter quantum estimation protocols, several variables are simultaneously evaluated, in a way which may outperform individual estimation strategies with equivalent resources [26,27], thereby motivating the use of such protocols in a variety of diverse contexts [26-29].

The use of peculiar quantum many-body state as a probe in quantum metrology can enhance the accuracy in parameter estimation [25,30]. Conversely, one may think of using quantum metrological tools in the study and characterisation of many-body systems. Noteworthy instances of many-body quantum systems are those experiencing quantum phase transitions. Indeed, quantum parameter estimation, with its intimate relation with geometric information, provides a novel and promising approach to investigate equilibrium [31-40] and out-of-equilibrium [41-48] quantum critical phenomena [49-54].

Fermionic Gaussian states (FGSs) play a major role in the derivation of exact and approximate solution of many-body problems of Fermionic and spin systems. Deriving closed form expressions 
of quantities involved in parameter estimation problems for many body quantum systems is a major challenge. This work addresses this task in the special, yet relevant, case of arbitrary FGSs.

Given a collection of parameters $\left\{\lambda_{\mu}\right\}_{\mu=1}^{N}$, a parameter estimation problem consists in finding an estimator $\hat{\lambda}=\hat{\lambda}\left(x_{1}, x_{2}, \ldots\right)$, i.e. a map from a set of measurement outcomes $\left(x_{1}, x_{2}, \ldots\right)$ to $\{\lambda\}$. In a quantum estimation protocol, $\lambda$ are a set of parameters labelling a quantum state. The quantum Fisher information matrix $J(\lambda)$ provides a figure of merit of the estimation precision of $\hat{\lambda}$, through the quantum Cramér-Rao bound (CRB) [1,19,55], i.e.,

$$
\operatorname{Cov}_{\lambda}[\hat{\lambda}] \geq J(\lambda)^{-1}
$$

which provides a lower bound (in matrix sense) on the covariance matrix $\operatorname{Cov}_{\lambda}[\hat{\lambda}]_{\mu \nu}=E_{\lambda}\left[(\hat{\lambda}-\lambda)_{\mu}(\hat{\lambda}-\lambda)_{v}\right]$. The Fisher information $J(\lambda)$ is defined as a matrix, whose entries are

$$
J_{\mu \nu}(\lambda)=\frac{1}{2} \operatorname{Tr} \rho\left\{L_{\mu}, L_{v}\right\},
$$

where $\left\{L_{\mu}\right\}$ is the symmetric logarithmic derivatives (SLD) with respect to the parameter $\lambda_{\mu}$. The SLD is an Hermitian operators, implicitly defined as the solution of the (continuous-time) Lyapunov equation

$$
\frac{\partial \rho}{\partial \lambda_{\mu}}=\frac{1}{2}\left(\rho L_{\mu}+L_{\mu} \rho\right)
$$

For single parameter estimation, the quantum Cramér-Rao bound (1) can always be saturated by choosing a measurement protocol which uses the projective measurement in the eigenbasis of the SLD. Indeed, the quantum Fisher information determines the ultimate achievable precision in estimating a single parameter in the asymptotic limit of an infinite number of experiment repetitions.

Unlike the single parameter case, in the multi-parameter scenario the CRB cannot always be saturated. Intuitively, this is due to the incompatibility of the optimal measurements for different parameters. A sufficient condition for the saturation is indeed $\left[L_{\mu}, L_{v}\right]=0$, which is however not a necessary condition. Within the comprehensive framework of quantum local asymptotic normality [56-59], a necessary and sufficient condition for the saturation of the multi-parameter CRB is given by [60]

$$
\mathcal{U}_{\mu v}=-\frac{i}{4} \operatorname{Tr} \rho\left[L_{\mu}, L_{v}\right]=0 \quad \forall \mu, v,
$$

and it is known as compatibility condition [60]. In the context of quantum information geometry, and quantum holonomies of mixed states, $\mathcal{U}_{\mu v}$ is known as mean Uhlmann curvature [52,53,61-64]. In this sense, $\mathcal{U}_{\mu \nu}$ marks the incompatibility between $\lambda_{\mu}$ and $\lambda_{\nu}$, and such an incompatibility arises from the inherent quantum nature of the underlying physical system.

We derive a closed form expression of the incompatibility condition and the SLD of FGSs, which are of fundamental importance in the analysis of steady-states of both equilibrium and non-equilibrium quantum many-body systems in their applications to quantum metrology.

Let's consider a systems of $n$ fermionic particles described by creation and annihilation operators $c_{j}^{\dagger}$ and $c_{j}$. FGSs are defined as states that can be expressed as

$$
\rho=\frac{e^{-\frac{i}{4} \omega^{T} \Omega \omega}}{Z}, \quad Z:=\operatorname{Tr}\left[e^{-\frac{i}{4} \omega^{T} \Omega \omega}\right]
$$

where $\Omega$ is a $2 n \times 2 n$ real antisymmetric matrix and $\omega:=\left(\omega_{1} \ldots \omega_{2 n}\right)^{T}$ is a 2 -dimensional array of Majorana Fermions, $\omega_{2 j-1}:=c_{j}+c_{j}^{\dagger}, \omega_{2 j}:=i\left(c_{j}-c_{j}^{\dagger}\right),\left\{\omega_{j}, \omega_{k}\right\}=2 \delta_{j k}$. Gaussian states are completely specified by the two-point correlation matrix

$$
\Gamma_{j k}:=1 / 2 \operatorname{Tr}\left(\rho\left[\omega_{j}, \omega_{k}\right]\right), \quad \Gamma=\Gamma^{\dagger}=-\Gamma^{T},
$$


which is equal to $\Gamma=\tanh \left(i \frac{\Omega}{2}\right)$.

One can show that $L$, for Gaussian states, is quadratic on Fermionic operators [53]. If this is the case, $L$ can be expressed as

$$
L=: \frac{1}{2} \omega^{T} \cdot K \cdot \omega+\zeta^{T} \cdot \omega+\eta,
$$

where $K:=\left\{K_{j k}\right\}_{j k=1}^{2 n}$ is $2 n \times 2 n$ Hermitian anti-symmetric matrix, $\zeta:=\left\{\zeta^{k}\right\}_{k=1}^{2 n}$ is a $2 n$ real vector, and $\eta$ is a real number. As for any odd-order correlation function, the mean of a Fermionic operator vanishes, i.e. $\left\langle\omega_{k}\right\rangle=\operatorname{Tr}\left(\rho \omega_{k}\right)=0, \forall k=1 \ldots 2 n$. By differentiating the former relation, one readily shows that the linear term in (7) is identically zero

$$
0=\frac{d}{d \lambda} \operatorname{Tr}\left(\rho \omega_{k}\right)=\operatorname{Tr}\left(\omega_{k} \dot{\rho}\right)=\frac{1}{2} \operatorname{Tr}\left(\omega_{k}\{L, \rho\}\right)=\operatorname{Tr}\left(\rho\left\{\zeta^{T} \omega, \omega_{k}\right\}\right)=\zeta^{k},
$$

where $\zeta^{k}$ is the $k$-th component of $\zeta$, and in the forth equality one takes into account that the third order correlations vanish. The trace preserving condition, $0=\frac{d}{d \lambda} \operatorname{Tr} \rho=\operatorname{Tr}(\dot{\rho})=\operatorname{Tr}(\rho L)$, leads to

$$
\eta=-\frac{1}{2} \operatorname{Tr}\left(\rho \omega^{T} K \omega\right)=\frac{1}{2} \operatorname{Tr}(K \Gamma) .
$$

In order to determine $K$, let's take differential of $\Gamma_{j k}=1 / 2 \operatorname{Tr}\left(\rho\left[\omega_{j}, \omega_{k}\right]\right)$, then

$$
\begin{aligned}
\dot{\Gamma}_{j k} & =\frac{1}{2} \operatorname{Tr}\left(\dot{\rho}\left[\omega_{j}, \omega_{k}\right]\right)=\frac{1}{4} \operatorname{Tr}\left(\{\rho, L\}\left[\omega_{j}, \omega_{k}\right]\right)=\frac{1}{8} \operatorname{Tr}\left(\left\{\rho, \omega^{T} K \omega\right\}\left[\omega_{j}, \omega_{k}\right]\right)+\frac{\eta}{4} \operatorname{Tr}\left(\rho\left[\omega_{j}, \omega_{k}\right]\right) \\
& =\frac{1}{8} \sum_{l m} K^{l m} \operatorname{Tr}\left(\left\{\rho,\left[\omega_{l}, \omega_{m}\right]\right\}\left[\omega_{j}, \omega_{k}\right]\right)+\frac{\eta}{2} \Gamma_{j k}=(\Gamma K \Gamma-K)_{j k}+\frac{1}{2}\left[\eta-\frac{1}{2} \operatorname{Tr}(K \Gamma)\right] \Gamma_{j k},
\end{aligned}
$$

where the last equality is obtained by using the antisymmetry of $\Gamma$ and $K$ under the exchange of $j$ and $k$ and with the help of Wick's theorem [65], i.e., $\operatorname{Tr}\left(\rho \omega_{k_{1}} \omega_{k_{2}} \ldots \omega_{k_{2 p}}\right)=\operatorname{Pf}\left(\Gamma_{k_{1} k_{2} \ldots k_{2 p}}\right), 1 \leq k_{1}<\ldots<k_{2 p} \leq 2 n$. Finally, according to Equation (8), the last term vanishes and we obtain the following (discrete time) Lyapunov equation

$$
\dot{\Gamma}=\Gamma K \Gamma-K,
$$

which can be formally solved by $K=\left(\operatorname{Ad}_{\Gamma}-\mathbb{1}\right)^{-1}(\dot{\Gamma})$, where $\operatorname{Ad}_{\Gamma}(X):=\Gamma X \Gamma^{\dagger}$ is the adjoint action. In the eigenbasis of $\Gamma$ (i.e., $\Gamma|j\rangle=\gamma_{j}|j\rangle$ ) it reads

$$
\langle j|K| k\rangle=(K)_{j k}=\frac{(\dot{\Gamma})_{j k}}{\gamma_{j} \gamma_{k}-1}=-\frac{\dot{\Omega}_{k}}{2} \delta_{j k}+\tanh \frac{\Omega_{j}-\Omega_{k}}{2}\langle j \mid \dot{k}\rangle,
$$

where, in the second equality, we made use of the relation $\gamma_{k}=\tanh \left(\Omega_{k} / 2\right)$, which yields the diagonal $(\dot{\Gamma})_{j j}=\left(1-\gamma_{j}^{2}\right) \dot{\Omega}_{j}$ and off-diagonal $(\dot{\Gamma})_{j k}=\left(\gamma_{k}-\gamma_{j}\right)\langle j \mid \dot{k}\rangle$ terms. Plugging the explicit expression of $L_{\mu}=\frac{1}{2} \omega^{T} \cdot K_{\mu} \cdot \omega+\frac{1}{2} \operatorname{Tr}\left(K_{\mu} \Gamma\right)$ into (2) produces the following results for the Fisher information matrix

$$
J_{\mu \nu}=\frac{1}{2} \operatorname{Tr}\left(K_{\mu} K_{v}-\Gamma K_{\mu} \Gamma K_{v}\right)=-\frac{1}{2} \operatorname{Tr}\left(\partial_{\mu} \Gamma K_{v}\right)=\frac{1}{2} \sum_{j k} \frac{\left(\partial_{\mu} \Gamma\right)_{j k}\left(\partial_{v} \Gamma\right)_{k j}}{1-\gamma_{j} \gamma_{k}},
$$

The above relation was derived by a different procedure by Banchi et al. [49]. The compatibility condition instead reads

$$
\mathcal{U}_{\mu v}=\frac{i}{4} \operatorname{Tr}\left(\Gamma\left[K_{\mu}, K_{v}\right]\right)=\frac{i}{4} \sum_{j k} \frac{\gamma_{k}-\gamma_{j}}{\left(1-\gamma_{j} \gamma_{k}\right)^{2}}\left(\partial_{\mu} \Gamma\right)_{j k}\left(\partial_{v} \Gamma\right)_{k j} .
$$

The expressions $(11,12,13)$, which are the main result of this work (cf. $[49,52,53])$, are well defined everywhere except for $\gamma_{j}=\gamma_{k}= \pm 1$, where the Gaussian state $\rho$ becomes singular (i.e., it is not 
full rank). In this condition, the expression (11) for the SLD L may become singular. Nevertheless, the boundness of the function $\left|\tanh \frac{\Omega_{j}-\Omega_{k}}{2}\right| \leq 1$ in (11) shows that such a singularity is relatively benign. One can readily check that this condition $\gamma_{j}=\gamma_{k}= \pm 1$ produces, at most, removable singularities in the Fisher information matrix (12) and on the compatibility condition (13) (cf. [66]). This allows the quantum Fisher information matrix, as well as the compatibility condition, to be extended by continuity from the set of full-rank density matrices to the subset with $\gamma_{j}=\gamma_{k}= \pm 1$.

\section{Conclusions}

In this work we derived a general expression for the incompatibility condition of an arbitrary Fermionic Gaussian state. We obtained a compact expression of the SLD in terms of correlation matrix of a FGS, which allows for the calculation of the quantum Fisher information and the compatibility condition in multi-parameter quantum estimation problems [60]. This provides a way of assessing the ultimate precision of parameter estimation problems in many-body systems involving Fermionic Gaussian states through the Cramer-Rao bound. Moreover, the expression of the SLD allows for the explicit derivation of the optimal quantum measurement needed for the parameter estimation of interest. Due to the quantum nature of the underlying probe, the multi-parameter estimation problem may not saturate the multi-parameter Cramer-Rao bound. Unlike classical estimation problems, the non-commutativity of the observables, involved in the optimal quantum measurements associated to independent parameters, may prevent the CRB from being saturated [22]. The explicit expression of the Fisher information together with the compatibility condition, in analogy to Bosonic Gaussian estimation problems [24], can be exploited, in combination with efficient numerical algorithms, to find optimal Fermionic Gaussian probe that minimises the overall multi-parameter estimation problem [67].

Author Contributions: A.C., B.S. and D.V. conceived the idea, interpreted the results and explained them. A.C. carried out calculations. All authors contributed to write and review the manuscript.

Acknowledgments: This work was supported by the Government of the Russian Federation through Agreement No. 074-02-2018-330(2). We acknowledge also partial support by Ministry of Education, University and Research of the Italian Government.

Conflicts of Interest: The authors declare no conflict of interest.

\section{References}

1. Helstrom, C.W. Quantum Detection and Estimation Theory; Academic Press: Cambridge, MA, USA, 1976.

2. Udem, T.; Holzwarth, R.; Hänsch, T.W. Optical frequency metrology. Nature 2002, 416, 233-237, doi:10.1038/416233a.

3. Katori, H. Optical lattice clocks and quantum metrology. Nat. Photonics 2011, 5, 203-210, doi:10.1038/nphoton.2011.45.

4. Giovannetti, V.; Lloyd, S.; Maccone, L. Quantum-enhanced measurements: beating the standard quantum limit. Science 2004, 306, 1330-1336, doi:10.1126/science.1104149.

5. Aspachs, M.; Adesso, G.; Fuentes, I. Optimal Quantum Estimation of the Unruh-Hawking Effect. Phys. Rev. Lett. 2010, 105, 151301, doi:10.1103/PhysRevLett.105.151301.

6. Ahmadi, M.; Bruschi, D.E.; Fuentes, I. Quantum metrology for relativistic quantum fields. Phys. Rev. D 2014, 89, 065028, doi:10.1103/PhysRevD.89.065028.

7. Schnabel, R.; Mavalvala, N.; McClelland, D.E.; Lam, P.K. Quantum metrology for gravitational wave astronomy. Nat. Commun. 2010, 1, 110-121, doi:10.1038/ncomms1122.

8. Aasi, J.; Abadie, J.; Abbott, B.P.; Abbott, R.; Abbott, T.D.; Abernathy, M.R.; Adams, C.; Adams, T.; Addesso, P.; Adhikari, R.X.; et al. Enhanced sensitivity of the LIGO gravitational wave detector by using squeezed states of light. Nat. Photonics 2013, 7, 613-619, doi:10.1038/nphoton.2013.177.

9. Correa, L.A.; Mehboudi, M.; Adesso, G.; Sanpera, A. Individual Quantum Probes for Optimal Thermometry. Phys. Rev. Lett. 2015, 114, 220405, doi:10.1103/PhysRevLett.114.220405. 
10. De Pasquale, A.; Rossini, D.; Fazio, R.; Giovannetti, V. Local quantum thermal susceptibility. Nat. Commun. 2016, 7, 1-8, doi:10.1038/ncomms12782.

11. Schmitt, S.; Gefen, T.; Stürner, F.M.; Unden, T.; Wolff, G.; Müller, C.; Scheuer, J.; Naydenov, B.; Markham, M.; Pezzagna, S.; Meijer, J.; Schwarz, I.; Plenio, M.; Retzker, A.; McGuinness, L.P.; Jelezko, F. Submillihertz magnetic spectroscopy performed with a nanoscale quantum sensor. Science 2017, 356, 832-837, doi:10.1126/science.aam5532.

12. Boss, J.M.; Cujia, K.S.; Zopes, J.; Degen, C.L. Quantum sensing with arbitrary frequency resolution. Science 2017, 356, 837-840, doi:10.1126/science.aam7009.

13. Tsang, M.; Nair, R.; Lu, X.M. Quantum Theory of Superresolution for Two Incoherent Optical Point Sources. Phys. Rev. X 2016, 6, 031033, doi:10.1103/PhysRevX.6.031033.

14. Nair, R.; Tsang, M. Far-Field Superresolution of Thermal Electromagnetic Sources at the Quantum Limit. Phys. Rev. Lett. 2016, 117, 190801, doi:10.1103/PhysRevLett.117.190801.

15. Lupo, C.; Pirandola, S. Ultimate Precision Bound of Quantum and Subwavelength Imaging. Phys. Rev. Lett. 2016, 117, 190802, doi:10.1103/PhysRevLett.117.190802.

16. Caves, C.M. Quantum-mechanical noise in an interferometer. Phys. Rev. D 1981, 23, 1693-1708, doi:10.1103/PhysRevD.23.1693.

17. Huelga, S.F.; Macchiavello, C.; Pellizzari, T.; Ekert, A.K.; Plenio, M.B.; Cirac, J.I. Improvement of Frequency Standards with Quantum Entanglement. Phys. Rev. Lett. 1997, 79, 3865-3868, doi:10.1103/PhysRevLett.79.3865.

18. Giovannetti, V.; Lloyd, S.; Maccone, L. Quantum metrology. Phys. Rev. Lett. 2006, 96, 010401, doi:10.1103/PhysRevLett.96.010401.

19. Paris, M.G.A. Quantum Estimation For Quantum Technology. Int. J. Quantum Inf. 2009, 7, 125-137, doi:10.1142/S0219749909004839.

20. Giovannetti, V.; Lloyd, S.; Maccone, L. Advances in quantum metrology. Nat. Photonics 2011, 5, 222-229, doi:10.1038/nphoton.2011.35.

21. Tóth, G.; Apellaniz, I. Quantum metrology from a quantum information science perspective. J. Phys. A Math. Theor. 2014, 47, 424006, doi:10.1088/1751-8113/47/42/424006.

22. Szczykulska, M.; Baumgratz, T.; Datta, A. Multi-parameter quantum metrology. Adv. Phys. X 2016, 1, 621-639, doi:10.1080/23746149.2016.1230476.

23. Pezzè, L.; Smerzi, A.; Oberthaler, M.K.; Schmied, R.; Treutlein, P. Quantum metrology with nonclassical states of atomic ensembles. Rev. Mod. Phys. 2018, 90, 035005, doi:10.1103/RevModPhys.90.035005.

24. Nichols, R.; Liuzzo-Scorpo, P.; Knott, P.A.; Adesso, G. Multiparameter Gaussian quantum metrology. Phys. Rev. A 2018, 98, 012114, doi:10.1103/PhysRevA.98.012114.

25. Braun, D.; Adesso, G.; Benatti, F.; Floreanini, R.; Marzolino, U.; Mitchell, M.W.; Pirandola, S. Quantum-enhanced measurements without entanglement. Rev. Mod. Phys. 2018, 90, 035006, doi:10.1103/RevModPhys.90.035006.

26. Humphreys, P.C.; Barbieri, M.; Datta, A.; Walmsley, I.A. Quantum enhanced multiple phase estimation. Phys. Rev. Lett. 2013, 111, 1-5, doi:10.1103/PhysRevLett.111.070403.

27. Baumgratz, T.; Datta, A. Quantum Enhanced Estimation of a Multidimensional Field. Phys. Rev. Lett. 2016, 116, 1-5, doi:10.1103/PhysRevLett.116.030801.

28. Pezzè, L.; Ciampini, M.A.; Spagnolo, N.; Humphreys, P.C.; Datta, A.; Walmsley, I.A.; Barbieri, M.; Sciarrino, F.; Smerzi, A. Optimal Measurements for Simultaneous Quantum Estimation of Multiple Phases. Phys. Rev. Lett. 2017, 119, 1-6, doi:10.1103/PhysRevLett.119.130504.

29. Apellaniz, I.; Urizar-Lanz, I.; Zimborás, Z.; Hyllus, P.; Tóth, G. Precision bounds for gradient magnetometry with atomic ensembles. Phys. Rev. A 2018, 97, 1-17, doi:10.1103/PhysRevA.97.053603.

30. Zanardi, P.; Paris, M.G.A.; Campos Venuti, L. Quantum criticality as a resource for quantum estimation. Phys. Rev. A 2008, 78, 042105, doi:10.1103/PhysRevA.78.042105.

31. Carollo, A.C.M.; Pachos, J.K. Geometric Phases and Criticality in Spin-Chain Systems. Phys. Rev. Lett. 2005, 95, 157203, doi:10.1103/PhysRevLett.95.157203.

32. Zhu, S.L. Scaling of Geometric Phases Close to the Quantum Phase Transition in the XY Spin Chain. Phys. Rev. Lett. 2006, 96, 077206, doi:10.1103/PhysRevLett.96.077206.

33. Hamma, A. Berry Phases and Quantum Phase Transitions. 2006. Available online: http://arxiv.org/abs/ quant-ph/0602091 (accessed on 15 April 2019). 
34. Zanardi, P.; Paunković, N. Ground state overlap and quantum phase transitions. Phys. Rev. E 2006, 74, 031123, doi:10.1103/PhysRevE.74.031123.

35. Campos Venuti, L.; Zanardi, P. Quantum Critical Scaling of the Geometric Tensors. Phys. Rev. Lett. 2007, 99, 095701, doi:10.1103/PhysRevLett.99.095701.

36. Campos Venuti, L.; Cozzini, M.; Buonsante, P.; Massel, F.; Bray-Ali, N.; Zanardi, P. Fidelity approach to the Hubbard model. Phys. Rev. B 2008, 78, 1-8, doi:10.1103/PhysRevB.78.115410.

37. Zanardi, P.; Giorda, P.; Cozzini, M. Information-Theoretic Differential Geometry of Quantum Phase Transitions. Phys. Rev. Lett. 2007, 99, 100603, doi:10.1103/PhysRevLett.99.100603.

38. Zanardi, P.; Campos Venuti, L.; Giorda, P. Bures metric over thermal state manifolds and quantum criticality. Phys. Rev. A 2007, 76, 062318, doi:10.1103/PhysRevA.76.062318.

39. Garnerone, S.; Jacobson, N.T.; Haas, S.; Zanardi, P. Fidelity Approach to the Disordered Quantum XY Model. Phys. Rev. Lett. 2009, 102, 057205, doi:10.1103/PhysRevLett.102.057205.

40. Rezakhani, A.T.; Abasto, D.F.; Lidar, D.A.; Zanardi, P. Intrinsic geometry of quantum adiabatic evolution and quantum phase transitions. Phys. Rev. A 2010, 82, 012321, doi:10.1103/PhysRevA.82.012321.

41. Magazzù, L.; Valenti, D.; Carollo, A.; Spagnolo, B. Multi-State Quantum Dissipative Dynamics in Sub-Ohmic Environment: The Strong Coupling Regime. Entropy 2015, 17, 2341-2354, doi:10.3390/e17042341.

42. Magazzú, L.; Carollo, A.; Spagnolo, B.; Valenti, D.; Magazzù, L.; Carollo, A.; Spagnolo, B.; Valenti, D. Quantum dissipative dynamics of a bistable system in the sub-Ohmic to super-Ohmic regime. J. Stat. Mech. Theory Exp. 2016, 2016, 54016, doi:10.1088/1742-5468/2016/05/054016.

43. Guarcello, C.; Valenti, D.; Carollo, A.; Spagnolo, B. Stabilization effects of dichotomous noise on the lifetime of the superconducting state in a long Josephson junction. Entropy 2015, 17, 2862-2875.

44. Spagnolo, B.; Valenti, D.; Guarcello, C.; Carollo, A.; Persano Adorno, D.; Spezia, S.; Pizzolato, N.; Di Paola, B. Noise-induced effects in nonlinear relaxation of condensed matter systems. Chaos, Solitons and Fractals 2015, 81, 412-424, doi:10.1016/j.chaos.2015.07.023.

45. Spagnolo, B.; Guarcello, C.; Magazzù, L.; Carollo, A.; Persano Adorno, D.; Valenti, D. Nonlinear Relaxation Phenomena in Metastable Condensed Matter Systems. Entropy 2016, 19, 20, doi:10.3390/e19010020.

46. Spagnolo, B.; Carollo, A.; Valenti, D. Enhancing Metastability by Dissipation and Driving in an Asymmetric Bistable Quantum System. Entropy 2018, 20, 226, doi:10.3390/e20040226.

47. Valenti, D.; Carollo, A.; Spagnolo, B. Stabilizing effect of driving and dissipation on quantum metastable states. Phys. Rev. A 2018, 97, 042109, doi:10.1103/PhysRevA.97.042109.

48. Spagnolo, B.; Carollo, A.; Valenti, D. Stabilization by dissipation and stochastic resonant activation in quantum metastable systems. Eur. Phys. J. Spec. Top. 2018, 227, 379-420, doi:10.1140/epjst/e2018-00121-x.

49. Banchi, L.; Giorda, P.; Zanardi, P. Quantum information-geometry of dissipative quantum phase transitions. Phys. Rev. E 2014, 89, 022102, doi:10.1103/PhysRevE.89.022102.

50. Marzolino, U.; Prosen, T. Quantum metrology with nonequilibrium steady states of quantum spin chains. Phys. Rev. A 2014, 90, 062130, doi:10.1103/PhysRevA.90.062130.

51. Kolodrubetz, M.; Sels, D.; Mehta, P.; Polkovnikov, A. Geometry and non-adiabatic response in quantum and classical systems. Phys. Rep. 2017, 697, 1-87, doi:10.1016/j.physrep.2017.07.001.

52. Carollo, A.; Spagnolo, B.; Valenti, D. Uhlmann curvature in dissipative phase transitions. Sci. Rep. 2018, 8, 9852, doi:10.1038/s41598-018-27362-9.

53. Carollo, A.; Spagnolo, B.; Valenti, D. Symmetric Logarithmic Derivative of Fermionic Gaussian States. Entropy 2018, 20, 485, doi:10.3390/e20070485.

54. Marzolino, U.; Prosen, T. Fisher information approach to nonequilibrium phase transitions in a quantum XXZ spin chain with boundary noise. Phys. Rev. B 2017, 96, 104402, doi:10.1103/PhysRevB.96.104402.

55. Holevo, A. Probabilistic and Statistical Aspects of Quantum Theory; Edizioni della Normale: Pisa, Italy, 2011; doi:10.1007/978-88-7642-378-9.

56. Hayashi, M.; Matsumoto, K. Asymptotic performance of optimal state estimation in qubit system. J. Math. Phys. 2008, 49, 102101, doi:10.1063/1.2988130.

57. Kahn, J.; Guţă, M.I. Local Asymptotic Normality for Finite Dimensional Quantum Systems. Commun. Math. Phys. 2009, 289, 597-652, doi:10.1007/s00220-009-0787-3. 
58. Gill, R.D.; Guţă, M.I. On asymptotic quantum statistical inference. In From Probability to Statistics and Back: High-Dimensional Models and Processes A Festschrift in Honor of Jon A. Wellner; Banerjee, M., Bunea, F., Huang, J., Koltchinskii, V., Maathuis, M.H., Ed.; Institute of Mathematical Statistics: Beachwood, OH, USA, 2013; Volume 9, pp. 105-127, doi:10.1214/12-IMSCOLL909.

59. Yamagata, K.; Fujiwara, A.; Gill, R.D. Quantum local asymptotic normality based on a new quantum likelihood ratio. Ann. Stat. 2013, 41, 2197-2217, doi:10.1214/13-AOS1147.

60. Ragy, S.; Jarzyna, M.; Demkowicz-Dobrzański, R. Compatibility in multiparameter quantum metrology. Phys. Rev. A 2016, 94, 052108, doi:10.1103/PhysRevA.94.052108.

61. Leonforte, L.; Valenti, D.; Spagnolo, B.; Carollo, A. Uhlmann number in translational invariant systems. Sci. Rep. 2019, 9, 9106, doi:10.1038/s41598-019-45546-9.

62. Bascone, F.; Leonforte, L.; Spagnolo, B.; Valenti, D.; Carollo, A. Finite temperature geometric properties of the Kitaev honeycomb model Phys. Rev. B 2019, 99, 205155, doi:10.1103/PhysRevB.99.205155.

63. Leonforte, L.; Valenti, D.; Spagnolo, B.; Dubkov, A.A.; Carollo, A. Haldane Model at finite temperature. J. Stat. Mech. Theory Exp. 2019, in Press.

64. Bascone F; Leonforte L; Valenti D; Spagnolo B and Carollo A. On critical properties of Berry curvature in Kitaev honeycomb model J. Stat. Mech. Theory Exp. 2019, in Press.

65. Bach, V.; Lieb, E.H.; Solovej, J.P. Generalized Hartree-Fock theory and the Hubbard model. J. Stat. Phys. 1994, 76, 3-89, doi:10.1007/BF02188656.

66. Šafránek, D. Discontinuities of the quantum Fisher information and the Bures metric. Phys. Rev. A 2017, 95, 052320, doi:10.1103/PhysRevA.95.052320.

67. Knott, P.A. A search algorithm for quantum state engineering and metrology. New J. Phys. 2016, 18, 073033, doi:10.1088/1367-2630/18/7/073033.

(C) 2019 by the authors. Licensee MDPI, Basel, Switzerland. This article is an open access article distributed under the terms and conditions of the Creative Commons Attribution (CC BY) license (http:/ / creativecommons.org/licenses/by/4.0/). 